\title{
Autophagy triggered by MAVS inhibits Coxsackievirus A16 replication
}

\author{
Y. SHI ${ }^{1,2}$, Y. LIU ${ }^{1,4}$, Y. ZHENG ${ }^{1}$, Y. TANG ${ }^{1}$, G. ZHU', W. QIU' ${ }^{2}$, L. HUANG ${ }^{1}$,

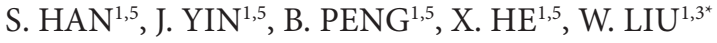

\begin{abstract}
${ }^{1}$ Hubei Province Key Laboratory of Allergy and Immunology, School of Basic Medical Sciences, Wuhan University, Wuhan 430071, P. R. China; ${ }^{2}$ Department of Immunology, School of Medicine, Jianghan University, Wuhan 430056, P. R. China; ${ }^{3}$ Shenzhen Research Institute, Wuhan University, Shenzhen 518057, P. R. China; ${ }^{4}$ Central Laboratory, Lianyungang First People's Hospital, Lianyungang 222002, P. R. China; ${ }^{5}$ Hubei Provincial Key Laboratory of Developmentally Originated Disease, Wuhan 430071, P. R. China; ${ }^{6}$ Department of Emergency, Wuhan General Hospital of the Chinese People's Liberation Army, Wuhan 430070, P. R. China
\end{abstract}

Received November 27, 2018; revised May 6, 2019; accepted September 9, 2019

\begin{abstract}
Summary. - Mitochondrial antiviral signaling protein (MAVS), a crucial adaptor protein localized on mitochondria, plays vital roles in various biological processes. Autophagy and apoptosis are two independent and closely linked cell death pathways. But whether MAVS could induce apoptosis and autophagy in rhabdomyosarcoma cells (RD cells) and what is the relationship between autophagy and apoptosis still remains elusive. Here, we reveal that overexpression of MAVS could trigger both apoptosis and autophagy in RD cells. Interestingly, MAVS-induced apoptosis was dependent on the activation of the c-Jun N-terminal kinase (JNK) signaling pathway and inhibition of the extracellular signal-regulated kinase (ERK) signaling pathway. Also, it was found that inhibition of autophagy by 3-methyladenine (3-MA) enhanced MAVS-induced apoptosis resulting in increased cleavage of caspase- 3 and poly (ADP-ribose) polymerase (PARP). Meanwhile, autophagy induction by rapamycin resulted in decreased MAVS-induced apoptosis. In addition, we found that MAVS expression was inhibited upon Coxsackievirus A16 (CA16) infection and overexpression of MAVS could inhibit CA16 replication. Collectively, our study provides novel insights into the link between apoptosis and autophagy induced by MAVS overexpression in RD cells and gains a greater understanding of MAVS-induced antiviral functions, which provide new targets for CA16 treatment.
\end{abstract}

Keywords: CA16; MAVS; apoptosis; autophagy

\section{Introduction}

MAVS, a crucial adaptor protein localized on mitochondria, consists of three structural and functional domains,

*Corresponding author. E-mail: liuwanhong@whu.edu.cn; phone: +86-27-68759985.

Abbreviations: 3-MA = 3-methyladenine; $\mathrm{Ab}=$ antibody; $\mathrm{CA} 16$ = Coxsackievirus A16; CARD $=\mathrm{N}$-terminal CARD-like domain; ERK = extracellular signal-regulated kinase; HFMD = hand, foot, and mouth disease; JNK = c-Jun N-terminal kinase; MAVS = mitochondrial antiviral signaling protein; MDA-5 = melanoma differentiation-associated gene 5 ; PARP $=$ poly (ADP-ribose) polymerase; $\mathrm{pi}$ = post-infection; $\mathrm{PRR}=$ proline-rich domain; $\mathrm{TM}=$ transmembrane domain namely the N-terminal CARD-like (CARD) domain, the middle proline-rich (PRR) domain, and the C-terminal transmembrane (TM) domain (Sun et al., 2006). The CARD-like domain of MAVS mediates its interaction with other CARD-containing proteins, such as RIG-I and MDA-5 (Akira et al., 2006; Kawai and Akira, 2006). MAVS not only interacts with RIG-I/MDA5 but also tumor necrosis factor receptor-associated factor 3 (TRAF3), which phosphorylates interferon regulatory factor $3 / 7$ (IRF-3/7), resulting in the production of cytokines such as type I IFN (IFN-I) (Akira et al., 2006; Kawai and Akira, 2006). Thus, MAVS functions as an adaptor which links dsRNA receptor RIG-I to the initiation of antiviral programs and mediates antiviral activity.

Apoptosis and autophagy are two of the most important cell death models (Kemp, 2017). Apoptosis is reported to be 
the first line of cellular defense against pathogen infection by activation of intracellular stress pathways, thereby resulting in cell death (Kemp, 2017). In contrast, autophagy is a homeostatic biological process of degrading and removing damaged or excess cellular organelles from the cytoplasm (Ohsumi, 2014). Although the two forms of programmed cell death utilize fundamentally distinct mechanisms, apoptosis and autophagy are highly interconnected (Kemp, 2017). The molecular mechanisms of crosstalk are beginning to be elucidated and have critical implications for the treatment of various diseases. Xie et al. (2014) found that $\beta$-catenin is involved in matrine-induced autophagy and apoptosis in WB-F344 cells. Ma et al. (2015) previously reported that overexpression of Homeobox containing 1 (HMBOX1) inhibited apoptosis induced by fibroblast growth factor 2 deprivation and promoted autophagy. But whether MAVS could induce apoptosis and autophagy in RD cells and what is the relationship between autophagy and apoptosis still remains largely unknown.

Coxsackievirus A16 (CA16) is a single-stranded, positivesense RNA virus and is one of the major causative agents of hand, foot, and mouth disease (HFMD) (Mao et al., 2014). HFMD is an infectious disease that is found worldwide, primarily affects infants and young children, and is characterized by mucocutaneous papulovesicular rashes on the hands, feet and mouth and buttocks lesions (Yin et al., 2018). In recent decades, numerous outbreaks of HFMD have occurred in the Asia-Pacific regions and neither an effective vaccine nor antiviral treatment is available (Jones et al., 2018). Therefore, it is of great importance to understand the pathogenesis of CA16 infection to develop strategies to better manage large outbreaks. Our previous studies revealed that CA16 infection triggers apoptosis in RD cells by inducing ER stress (Zhu et al., 2013). Meanwhile, we also demonstrated that CA16 could elicit autophagy involving the mTOR and
ERK pathways. MAVS has been reported to be associated with various virus infections, such as hepatitis $C$ virus, coxsackievirus B3, infectious bronchitis virus, and so on (Qin et al., 2017; Yu et al., 2017; Mukherjee et al., 2011). But the relationship between CA16 infection and MAVS expression still remains unknown.

In the present study, we report that overexpression of MAVS can induce apoptosis and autophagy in RD cells. Moreover, MAVS inhibited CA16 replication.

\section{Materials and Methods}

Cells, viruses and plasmids. RD (rhabdomyosarcoma) cell line was purchased from the American Type Culture Collection (ATCC). RD cells were maintained in minimum essential medium (MEM) supplemented with $10 \%$ fetal bovine serum (FBS) (PANbiotech, Germany) or $2 \%$ FBS (maintenance medium). The cells were maintained at $37^{\circ} \mathrm{C}$ and $5 \% \mathrm{CO}_{2}$. CA16 is a laboratory strain that has been completely sequenced and belongs to the $\mathrm{B} 1$ genotype (Shi et al., 2015, 2016). Plasmids expressing the His-tagged fulllength MAVS and deletion mutants lacking either the CARD-like domain $(\triangle \mathrm{CARD})$ or the transmembrane domain $(\triangle \mathrm{TM})$ were amplified by PCR and cloned into the pcDNA 6/V5-His B vector (Thermo Fisher Scientific, USA). The PCR primers used in this study are summarized in Table 1.

Reagents and antibodies. SP600125 (a JNK inhibitor) and PD98059 (a non-ATP competitive MEK inhibitor) were purchased from Selleck (USA). 3-methyladenine (3-MA) and Rapamycin (Rapa) were obtained from Sigma (USA). The rabbit anti-LC3B, anti-p62/SQSTM1, anti-PARP, anti- caspase-3, anti-cleaved caspase-3, non-phospho- or phospho-JNK and ERK1/2 polyclonal antibodies $(\mathrm{Ab})$ were purchased from Cell Signaling Technology (USA). The anti-actin Ab was purchased from Abcam (UK). The rabbit anti-MAVS Ab was obtained from Proteintech (USA). One

Table 1. Primers used for the construction of various plasmids and qRT-PCR

\begin{tabular}{lll}
\hline Primer name & Sequence (5'-3') & Purpose \\
\hline GAPDH-QPCR-F & AATGGAAATCCCATCACCATCT & For real-time polymerase chain reaction (qPCR) \\
GAPDH-QPCR-R & CGCCCCACTTGATTTTGG & \\
MAVS-F & GGGTACCATGCCGTTTGCTGAAGACA & Cloning MAVS sequence for pcDNA6.0-V5-HisB-MAVS \\
MAVS-R & GCTCTAGAGAGTGCAGACGCCGCCGGT & \\
MAVS- $\Delta$ CARD-F & GGGTACCATGGGCTGTGAGCTAGTTGATCT & Cloning MAVS sequence for pcDNA6.0/V5-HisB-MAVS $\Delta$ CARD \\
MAVS- $\Delta$ CARD-R & GCTCTAGAGAGTGCAGACGCCGCCGGT & \\
MAVS- $\triangle$ TM-F & GGGTACCATGCCGTTTGCTGAAGACA & Cloning MAVS sequence for pcDNA6.0/V5-HisB-MAVS $\Delta$ TM \\
MAVS- $\triangle$ TM-R & GCTCTAGAGACCCAGGTGAGGGCCTGTGGCA & \\
CA16-QPCR-F & ATTGGTGCTCCCACTACAGC & For real-time polymerase chain reaction (qPCR) \\
CA16-QPCR-R & TCAGTGTTGGCAGCTGTAGG & \\
MAVS-QPCR-F & CCCTCTGGCATCTCTTCAATAC & For real-time polymerase chain reaction (qPCR) \\
MAVS-QPCR-R & GCGAGATCAACTAGCTCACAG & \\
\hline
\end{tabular}

Bold and italics, restriction endonuclease cutting sites. F: forward primer; R: reverse primer. 
human polyclonal anti-CA16 serum sample was also used. The MAVS shRNAs and the control scrambled shRNA were designed and synthesized by Shanghai Longqian Bio-Technique Co. Ltd. (http://www.lqbiotech.com, China).

Viral infection, drug treatment and cell viability assay. $\mathrm{RD}$ cells were seeded into 6-well or 12-well plates and cultured until $80 \%$ confluency. The cells were then infected with CA16 at a multiplicity of infection (MOI) of 1 . After $1 \mathrm{~h}$, the cells were washed three times with phosphate-buffered saline (PBS) to remove unattached viruses and incubated in maintenance medium $(2 \% \mathrm{FBS})$ at $37^{\circ} \mathrm{C}$ for the indicated times. RD cells were pretreated with optimal concentrations of SP600125 (1.5 $\mu \mathrm{M})$ or Rapa $(100 \mathrm{nM})$ for $4 \mathrm{~h}$ and then transfected with empty vector or MAVS expression plasmids. For PD98059 $(2 \mu \mathrm{M})$ and 3-MA $(10 \mathrm{mM})$ treatment, cells were pretreated for $2 \mathrm{~h}$ and then transfected with the indicated plasmids. The MTT assay kit (Beyotime, China) was used to evaluate cell viability as described previously (Zhu et al., 2013).

Western blot analysis. Cells were washed twice with PBS and lysed on ice with RIPA buffer (Beyotime, China) containing $1 \times$ protease inhibitor cocktail, and protein concentrations were measured with the BCA protein assay (Thermo Fisher Scientific, USA). Then, the lysates were centrifuged for $10 \mathrm{~min}$ at 13,000 rpm at $4^{\circ} \mathrm{C}$ to clear cellular extract. The samples were boiled at $100^{\circ} \mathrm{C}$ for $5 \mathrm{~min}$ in sample buffer ( $2 \%$ SDS, $10 \%$ glycerol, $60 \mathrm{mM}$ Tris [pH 6.8], 5\% $\beta$-mercaptoethanol, and $0.01 \%$ bromophenol blue) before electrophoretic separation. Protein samples were separated by SDS-PAGE and transferred onto polyvinylidene fluoride (PVDF) membranes (Millipore, USA). Nonspecific antibody binding sites were blocked with $5 \%$ nonfat milk powder for $2 \mathrm{~h}$ at room temperature. The membranes were then bound with primary antibodies overnight at $4^{\circ} \mathrm{C}$ followed by horseradish peroxidase (HRP)-conjugated secondary antibody (Tianjin Sungene Biotech Co, Ltd, China) for $1.5 \mathrm{~h}$ at room temperature. The signals were detected by enhanced chemiluminescence using a commercial kit (Pierce, USA) according to the manufacturer's protocols. The band intensities were quantified using Image J software (NIH, USA).

RNA extraction and real-time PCR. According to the manufacturer's guidelines, total RNA was prepared with TRIzol (Invitrogen, USA). Then, $2 \mu \mathrm{g}$ of total RNA was reverse transcribed into $20 \mu \mathrm{l}$ cDNA using Reverse Transcription kit (Thermo Fisher Scientific, USA) according to the manufacturer's protocol. The cDNA samples were subjected to real-time PCR (Bio-Rad iQ5, USA) analysis using SYBR Green dye (Gene Copoeia Inc., USA). GAPDH was used as an internal control. The primer sets used for real-time PCR are shown in Table 1. To determine the MAVS and CA16 mRNA levels, the following conditions were used: $94^{\circ} \mathrm{C}$ for $5 \mathrm{~min}$, followed by 40 cycles of $94^{\circ} \mathrm{C}$ for $10 \mathrm{~s}, 55-60^{\circ} \mathrm{C}$ for $20 \mathrm{~s}$ and $72^{\circ} \mathrm{C}$ for $30 \mathrm{~s}$. All samples were run in triplicate and the data were analyzed using the $2^{-\Delta \Delta \mathrm{Ct}}$ method.

RNA interference. RD cells were plated in 6-well or 12-well plates $24 \mathrm{~h}$ prior to transfection. When the cells reached $50 \%$ confluency, they were transiently transfected with MAVS shRNAs using
Lipofectamine 2000 (Invitrogen) according to the manufacturer's instructions. A scrambled shRNA was used as the negative control. The silencing efficiency was determined by western blot analysis using the respective antibodies.

Confocal fluorescence microscopy. RD cells were seeded in 12- or 24-well plates that contained coverslips and grown to $70 \%$ confluence. Then, RD cells were transfected with GFP-LC3, pcDNA6.0 or MAVS plasmids using Lipofectamine 2000 (Invitrogen) according to the manufacturer's guidelines. The cells were transfected with plasmids and treated with drugs as described above at $24 \mathrm{~h}$ posttransfection. Treated cells were washed twice with PBS and fixed in $4 \%$ paraformaldehyde in PBS for 15 min. Coverslips were inverted onto slides containing $50 \%$ glycerol, and fluorescence signals were visualized with a confocal fluorescence microscope (FV1000S, Olympus, Japan).

$50 \%$ tissue culture infective doses $\left(T_{C I D} D_{50}\right.$. The $50 \%$ tissue culture infective doses $\left(\mathrm{TCID}_{50}\right.$ ) were determined as previously described (Shi et al., 2015). Briefly, RD cells were infected with virus, seeded in 96 -well plates, and then incubated at $37^{\circ} \mathrm{C}$ for 1 week to observe the cytopathic effect (CPE). The $\mathrm{TCID}_{50}$ was calculated following the method of Reed and Muench (Stanic, 1963).

Statistical analysis. Data were expressed as the means \pm standard deviations. Statistical analyses were performed using GraphPad Prism (GraphPad Software, USA) to evaluate the differences between experimental groups. Statistical significance was determined using Student's $t$-test and expressed as $\mathrm{P}$-values. $\mathrm{P}<0.05$ was considered to be statistically significant.

\section{Results}

\section{MAVS induces apoptosis in RD cells}

To determine whether MAVS could induce apoptosis in $\mathrm{RD}$ cells, we constructed plasmid containing MAVS and transfected it into RD cells. As shown in Fig. 1b, c, overexpression of MAVS significantly decreased cell viability and caused the RD cells to become round and lose adherence. It was shown that the overexpression of MAVS increased the levels of cleaved PARP and cleaved caspase- 3 by western blot analysis.

MAVS consists of three structural and functional domains (CARD, PRR, TM) and we generated MAVS mutant plasmids lacking either the CARD-like domain $(\triangle \mathrm{CARD})$ or the transmembrane domain $(\triangle T M$, Fig. 1a) to ascertain the roles of these domains in MAVS-induced apoptosis. We transfected the two truncation mutants into RD cells, and analyzed cell viability by MTT assay. As shown in Fig. 1b, cell death in the groups transfected with the two truncation mutants was higher than that of the mock transfected groups but lower than that of the MAVS transfected group. This result was further supported by Fig. 1c, which showed the change of morphology and adhesion, meaning that transfection of 
(a)

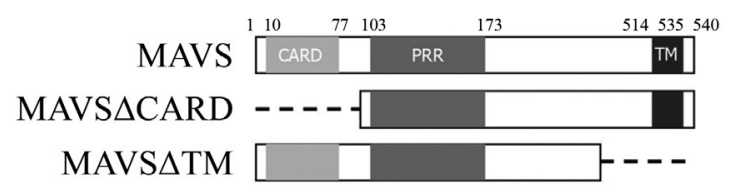

(b)

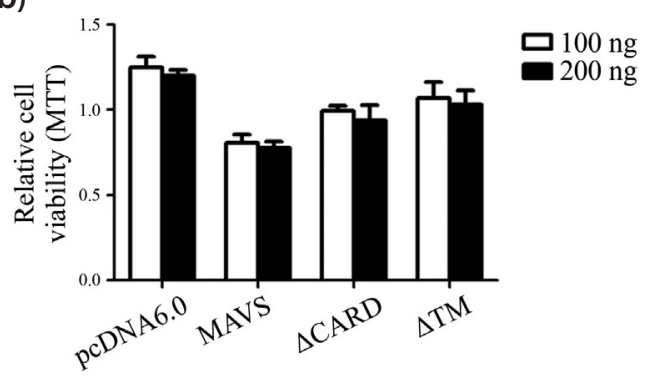

(c)
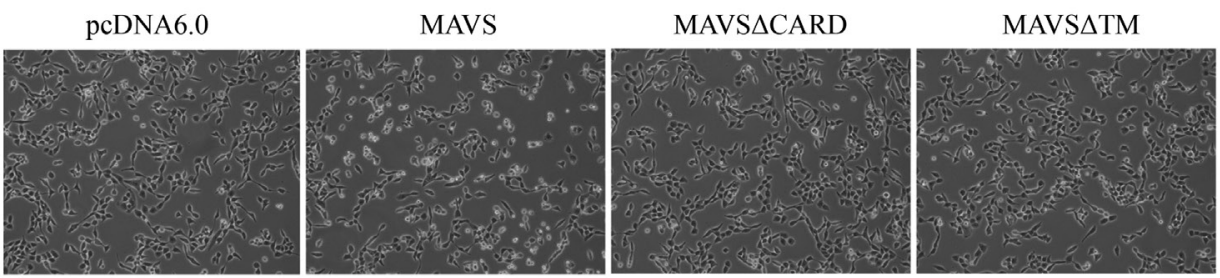

(d)
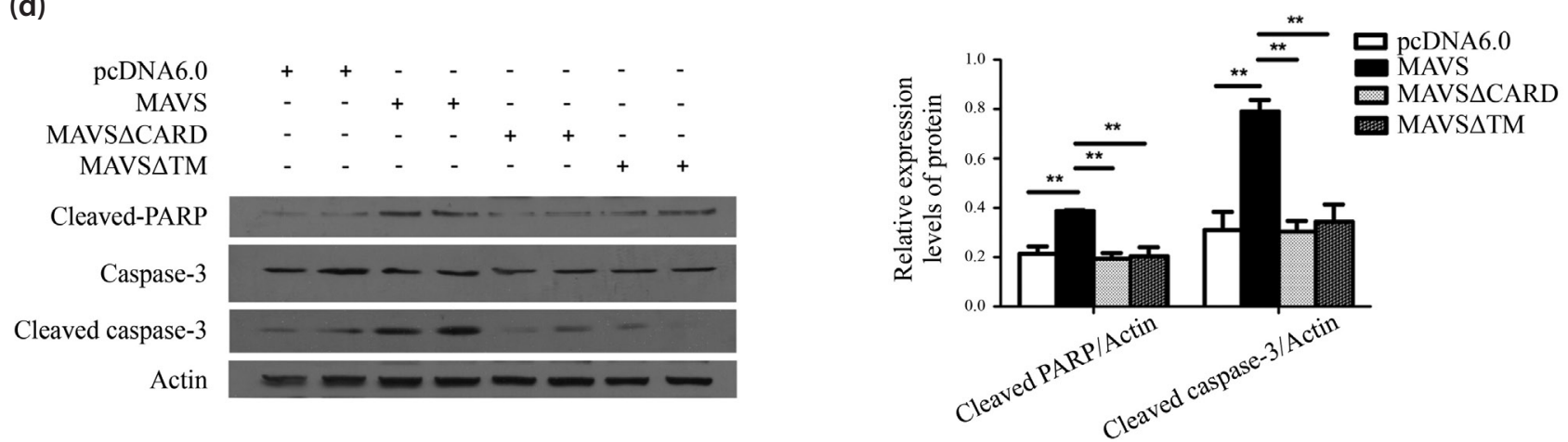

Fig. 1

Distinct MAVS domains are required for apoptosis induction

(a). The domain structure of MAVS. MAVS contains a N-terminal CARD (aa 10 to 77), a C-terminal transmembrane domain (TM, aa 514 to 535) and a central proline-rich region (PRR, aa 103 to 173). The cartoon shows the structure of the three truncation mutants used in the study. (b) The MTT assay was performed to determine the cell viability of RD cells transfected with pcDNA6.0-myc/HisB-MAVS or MAVS mutant plasmids (24 h post-transfection). (c) The morphology of the transfected RD cells was investigated by microscopy. RD cells were transfected with full-length MAVS gene or two truncation mutants, and cell morphology was observed $24 \mathrm{~h}$ post-transfection. (d) RD cells were transfected with full-length MAVS gene or two truncation mutants and cells were lysed $24 \mathrm{~h}$ later. The cell lysates were analyzed by SDS-PAGE, followed by western blot analysis using the indicated antibodies. Data are representatives of those from at least 3 independent experiments.

the truncation mutants inhibited apoptosis to some extent. As shown in western blot assays, deletion of the CARD-like domain or the transmembrane domain abolished the ability of MAVS to induce apoptosis, as confirmed by lower levels of cleaved PARP and cleaved caspase-3 expression (Fig. 1d). Taken together, these results suggest that overexpression of MAVS induces apoptosis in RD cells and both the CARDlike domain and the transmembrane domain contribute to apoptosis induction.
MAVS-induced apoptosis depends on the JNK and ERK signal pathways

The JNK and ERK cascades are evolutionarily conserved pathways that play important roles in various biological processes, including apoptosis (Li et al., 2014; Dhanasekaran and Reddy, 2008). To explore whether the JNK and ERK cascades are involved in the MAVS-induced apoptosis, we evaluated the phosphorylation of these kinases. As demonstrated in Fig. 2a, overexpression of MAVS induced 
(a)

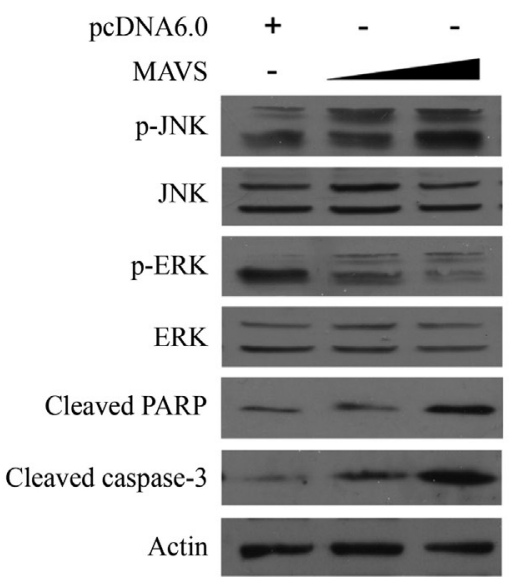

(b)

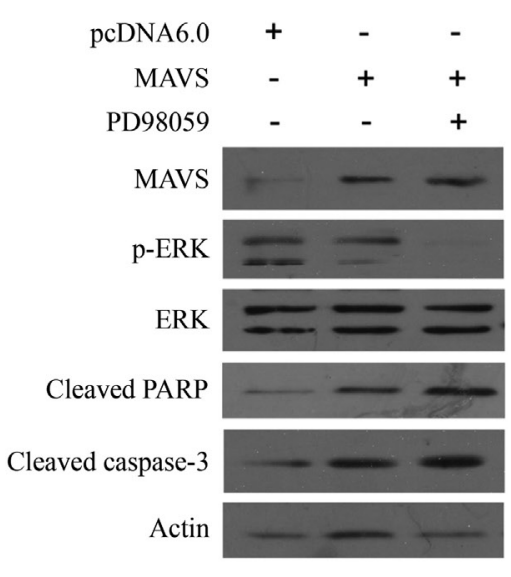

(c)

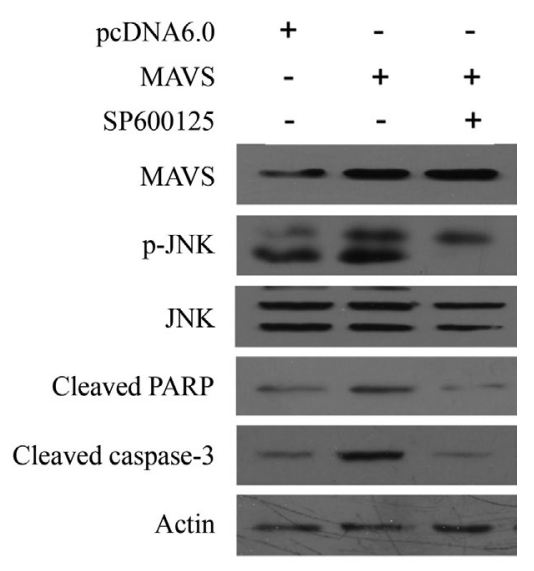

Fig. 2

MAVS partly modulates apoptosis by inhibiting the ERK signaling pathway and activating the JNK signaling pathway (a) RD cells were transfected with the pcDNA6.0-myc/HisB-MAVS plasmid or empty pcDNA6.0 vector at increasing concentrations $(0,1$ or $2 \mathrm{mg} /$ well in a 12-well plate) for $24 \mathrm{~h}$, and cell lysates were then collected for western blot analysis using the indicated antibodies. (b) RD cells were pretreated with or without an ERK inhibitor (PD98059, $2 \mu \mathrm{M}$ ) for $2 \mathrm{~h}$, followed by transfection with the pcDNA6.0-myc/HisB-MAVS plasmid or empty pcDNA6.0 vector. At $24 \mathrm{~h}$ after transfection, the cells were subjected to western blot analysis with the indicated antibodies. (c) RD cells were pretreated with or without a JNK inhibitor (SP600125, $1.5 \mu \mathrm{M}$ ) for $4 \mathrm{~h}$, followed by transfection with the pcDNA6.0-myc/HisB-MAVS plasmid or empty pcDNA6.0 vector. At $24 \mathrm{~h}$ after transfection, the cells were subjected to western blot analysis with the indicated antibodies. Actin was used as the loading control. Data are presented as the means from three independent experiments.

apoptosis and decreased the levels of ERK phosphorylation. In addition, the MEK inhibitor PD98059 was used to test whether the apoptosis induction was dependent on ERK inhibition (Fig. 2b). As expected, disrupting p-ERK with PD98059 enhanced the apoptosis response. Meanwhile, we examined p-JNK levels in response to apoptotic stimuli after MAVS transfection to clarify the role of JNK in the induction of apoptosis. As shown in Fig. 2a, overexpression of MAVS activated the phosphorylation of JNK at $24 \mathrm{~h}$ posttransfection but had no obvious effect on total JNK levels in RD cells. To further explore whether JNK signaling was involved in apoptosis induction, the cells were pretreated with a JNK inhibitor (SP600125). As expected, the levels of phosphorylated JNK were decreased and the levels of cleaved PARP and caspase-3 were decreased after SP600125 treatment (Fig. 2c). Taken together, our results indicate that MAVS may trigger apoptosis by activating the JNK and inhibiting ERK signaling pathways.

\section{Overexpression of MAVS triggers autophagy in RD cells}

It has been previously shown that MAVS preferentially forms a complex with the phosphatidylethanolamineconjugated form of microtubule-associated protein light chain 3 (LC3-II) (Sun et al., 2016). To further illuminate whether MAVS could induce autophagy in RD cells, we first performed western blot analysis to detect the expression of
LC3, which is a widely used hallmark of autophagy (Yoshii and Mizushima, 2017). As shown in Fig. 3a, overexpression of MAVS enhanced conversion of LC3-I to LC3-II and decreased the levels of p62/SQSTM1. Another good marker of autophagy activation is the punctate accumulation of LC3, which represents the recruitment of LC3-II to autophagic vacuoles (Yoshii and Mizushima, 2017). To further demonstrate that overexpression of MAVS can increase autophagosome formation, we investigated GFP-LC3 dot formation. Compared with control cells, large numbers of punctate GFP-LC3 proteins were observed in MAVS-transfected cells. Similar results were also observed in the rapamycintreated group $(\mathrm{P}<0.01)$ (Fig. 3b). These results showed that overexpression of MAVS could induce the formation of autophagosomes.

\footnotetext{
Inhibition of autophagy enhances MAVS- induced apoptosis

To detect the relationship between apoptosis and autophagy induced by MAVS, we tested whether autophagy inhibition can influence apoptosis by using 3-methyl adenine (3-MA), a conventional inhibitor of autophagy. $\mathrm{RD}$ cells were pretreated with 3-MA and then transfected with pcDNA6.0 plasmid or MAVS plasmid for an additional $24 \mathrm{~h}$. As shown in Fig. 4, the levels of LC3-II protein were decreased after 3-MA treatment in the MAVS plasmid groups
} 
(a)

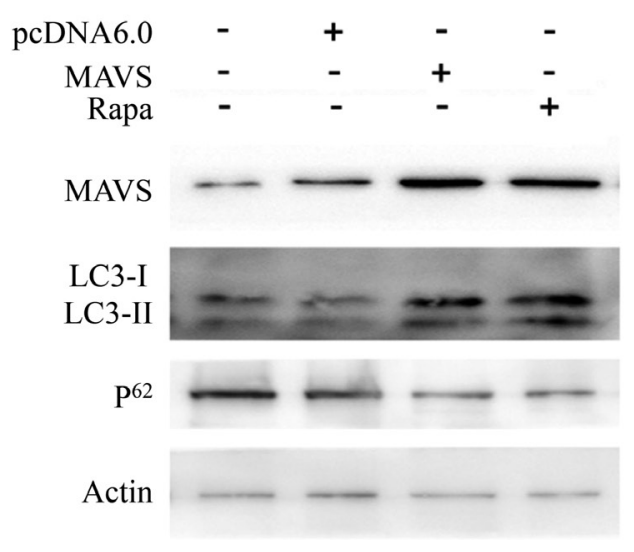

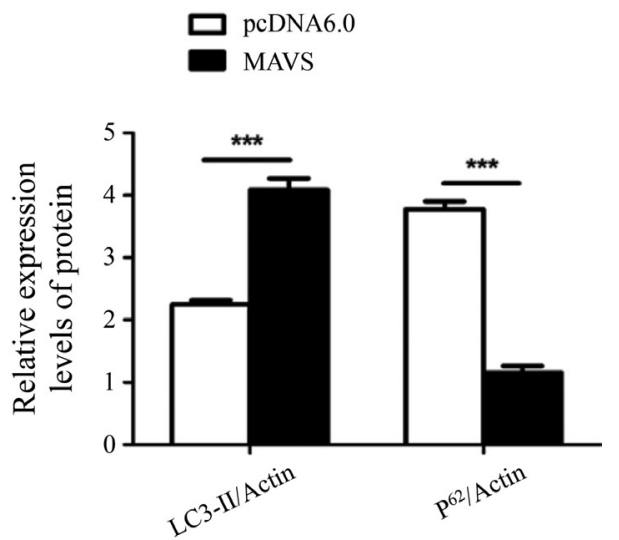

(b)

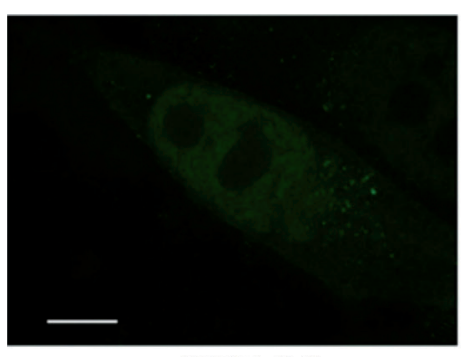

pcDNA6.0

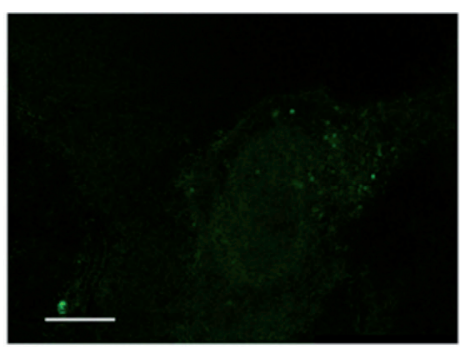

MAVS

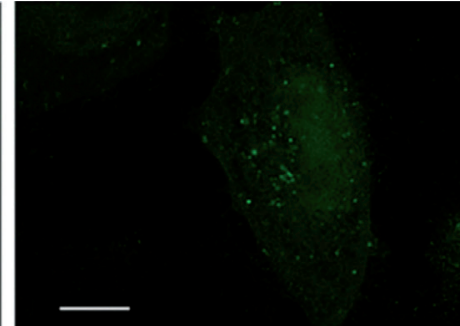

Rapa

Fig. 3

Overexpression of MAVS triggers autophagy in RD cells

(a) Western blot analysis of LC3 protein expression in RD cells transfected with MAVS. RD Cells were transfected with pcDNA6.0 or pcDNA6.0-myc/ HisB-MAVS. After 24 h, cells were harvested and detected with anti-LC3B and p62 antibodies; the results were compared to control cells. (b) GFP-LC3 dots were visualized via confocal microscopy. RD cells were transfected with GFP-LC3, pcDNA6.0 or MAVS plasmids for 24 h or treated with Rapamycin. Representative images are shown. Scale bar, $2 \mu \mathrm{m}$.

than in the control groups. Meanwhile, the levels of cleaved PARP and caspase-3 were increased after 3-MA treatment. These results show that autophagy inhibition may promote MAVS-induced apoptosis.

\section{Induction of autophagy reduces MAVS- induced} apoptosis

To further define the relationship between autophagy and apoptosis, $\mathrm{RD}$ cells were pretreated with rapamycin (Rapa) which is a widely used autophagy inducer. RD cells were pretreated with $100 \mu \mathrm{M}$ Rapa and then transfected with pcDNA6.0 plasmid or MAVS plasmid for an additional 24 h. As shown in Fig. 5, the conversion of LC3-I to LC3-II increased upon Rapa treatment in the MAVS-transfected group as compared with the control group. Meanwhile, the levels of cleaved PARP and caspase- 3 were decreased upon Rapa treatment in the experimental group compared with controls. Overall, these results indicated that autophagy induction decreased MAVS-induced apoptosis.
MAVS expression was decreased during CA16 infection and overexpression of MAVS inhibited CA16 replication

Previous studies have shown that MAVS is associated with various virus infections by eliciting innate immunity response. But until now, the relationship between MAVS expression and CA16 infection still remains largely unknown. To better understand the molecular mechanisms, we examined the effect of CA16 infection on MAVS expression. As shown in Fig. 6a, CA16 infection triggered decrease in the mRNA levels of MAVS, which was demonstrated by realtime PCR analysis (Fig. 6a). In addition, the protein levels of MAVS also decreased upon CA16 infection (Fig. 6b).

To further investigate the role of MAVS in CA16 replication, RD cells were transiently transfected with His-MAVS to overexpress MAVS. As depicted in Fig. 6c, the MAVS protein was successfully overexpressed in RD cells as detected by real-time PCR and western blot analysis. In addition, the levels of CA16 replication decreased significantly in the MAVS overexpression groups compared with the control 

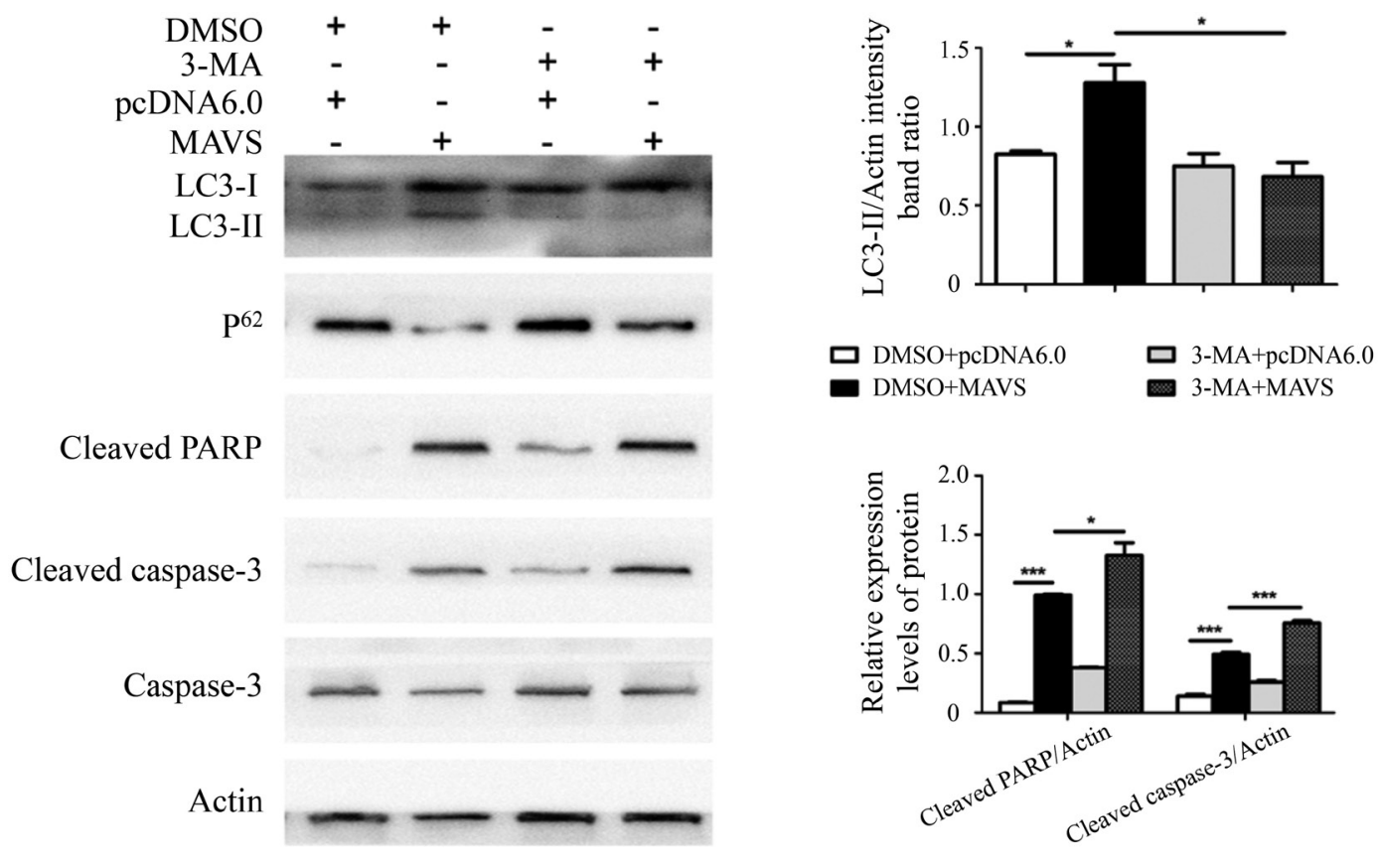

Fig. 4

Inhibition of autophagy enhances MAVS- induced apoptosis

Western blot analysis of cells inhibited by 3-MA. RD cells were pretreated with 3-MA for $4 \mathrm{~h}$, followed by transfection with pcDNA6.0 or MAVS. At $24 \mathrm{~h}$ after transfection, the cells were subjected to western blot analysis using anti-LC3B, p62, cleaved PARP, cleaved caspase- 3 and caspase- 3 antibodies. Significance was analyzed with a two-tailed Student's $t$ test. ${ }^{*} P<0.05,{ }^{* *} P<0.01,{ }^{* *} P<0.001$.
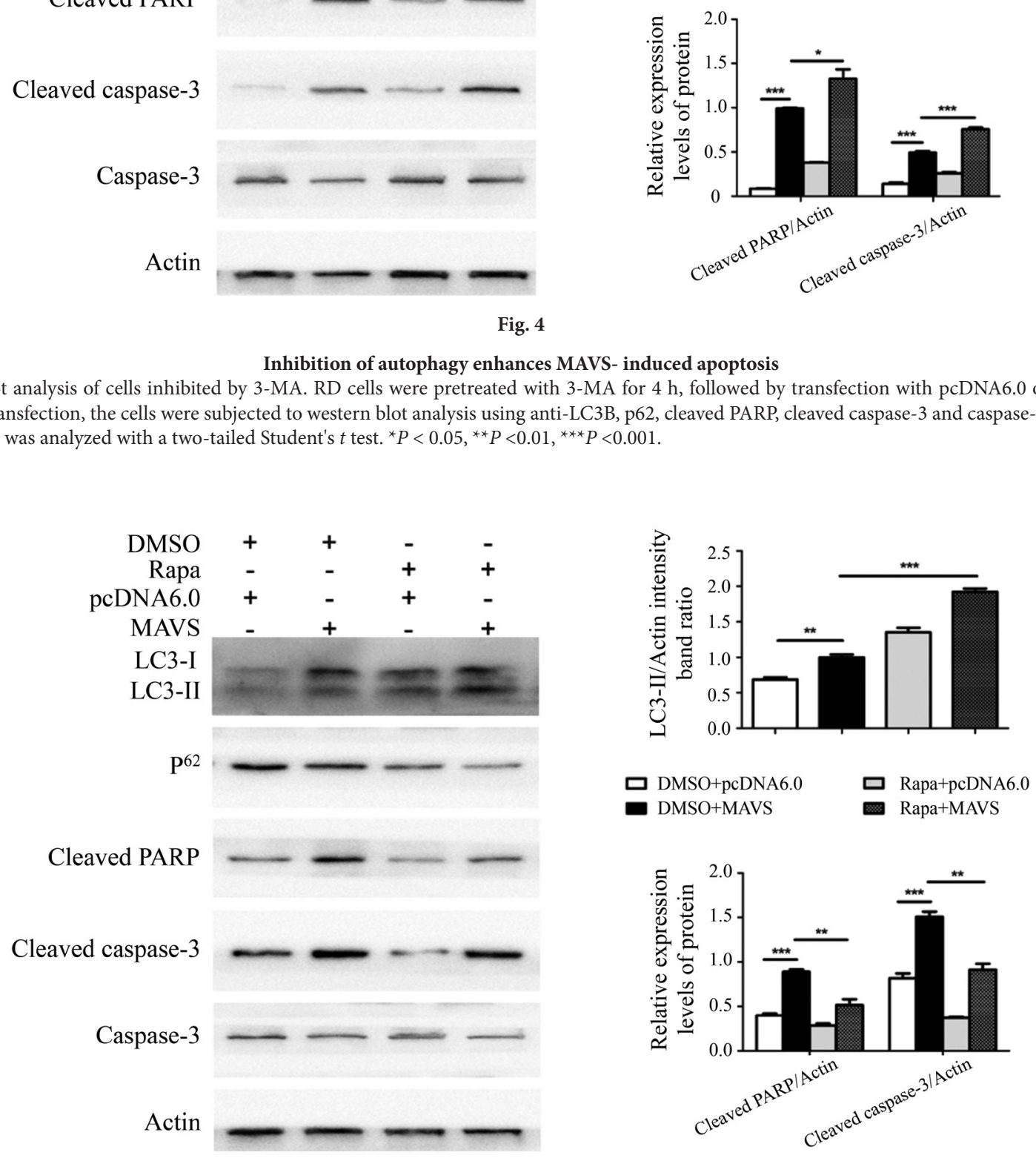
(a)

(b)

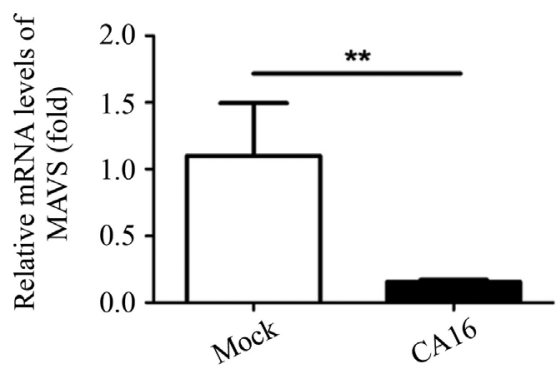

(d)

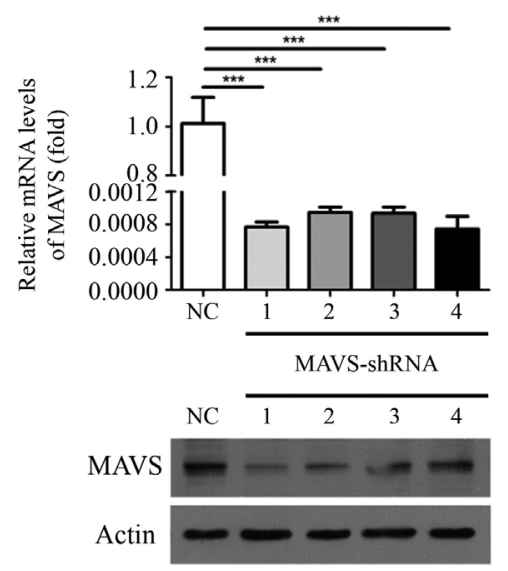

(c)

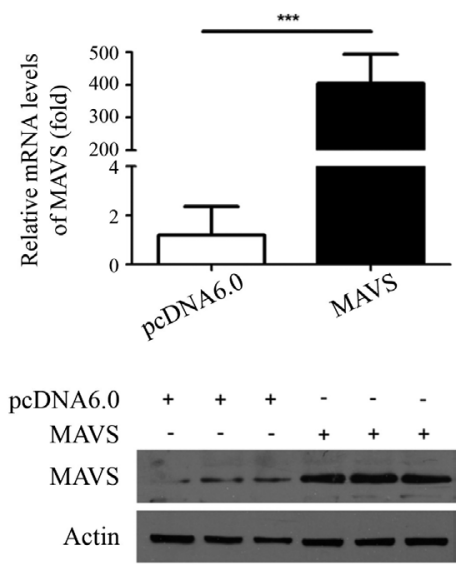

(g)

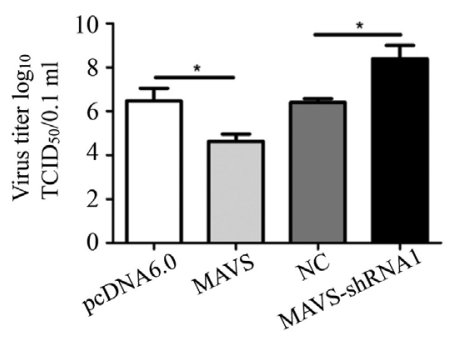

CA16 infection decreased MAVS expression and overexpression of MAVS inhibited CA16 replication

(a) Real-time PCR analysis of MAVS mRNA levels in RD cells that were infected with CA16 or uninfected. The cells were infected with CA16 at MOI of 1. After $1 \mathrm{~h}$ of virus absorption at $37^{\circ} \mathrm{C}$, the cells were further cultured in maintenance medium. The cells were harvested at $24 \mathrm{~h}$ pi and MAVS expression was detected with MAVS specific primers $\left(\mathrm{n}=3,{ }^{\star} P<0.05\right)$. (b) Western blot analysis of MAVS protein levels in RD cells that were infected with CA16 or uninfected. Three independent experiments were performed and shown. (c) Real-time PCR and western blot analysis of MAVS levels in RD cells. The cells were transfected with the empty vector (pcDNA6.0) or pcDNA6.0-HisB-MAVS for $24 \mathrm{~h}$, and cell lysates were then collected for western blot analysis using MAVS antibody. Meanwhile, the MAVS mRNA levels were detected by real-time PCR $\left({ }^{* * *} P<0.001\right)$. Three independent experiments were performed and shown. (d) Real-time PCR and western blot analysis of MAVS levels in RD cells that were transfected or not with MAVS-shRNA plasmids (MAVS-shRNA1, MAVS-shRNA2, MAVS-shRNA3, MAVS-shRNA4). The cells were transfected with an shRNA negative control or MAVS-shRNA plasmids for $48 \mathrm{~h}$, and the cell lysates were then collected for western blot analysis using MAVS antibodies. Meanwhile, the MAVS mRNA levels were detected by real-time PCR. ${ }^{* *} P<0.001$. (e) Overexpression of MAVS inhibited CA16 replication. RD cells were transfected with empty vector, pcDNA6.0-myc/ HisB-MAVS or MAVS mutant plasmids for $24 \mathrm{~h}$, followed by infection with CA16 (MOI = 1). At $12 \mathrm{~h}$ pi with CA16, the cell lysates were subjected to real-time PCR analysis using CA16-specific primers. ${ }^{\star} P<0.05$. (f) Depletion of MAVS expression enhanced CA16 replication. RD cells were transfected with an shRNA negative control or MAVS-shRNA plasmids (MAVS-shRNA1, MAVS-shRNA2) for $48 \mathrm{~h}$, followed by infection with CA16 (MOI $=1)$. At $12 \mathrm{~h} \mathrm{pi}$, the cell lysates were subjected to real-time PCR analysis using CA16-specific primers. Significance was analyzed with a two-tailed Student's $t$ test. ${ }^{*} P<0.05$. (g) The $50 \%$ tissue culture infective dose $\left(\mathrm{TCID}_{50}\right)$ values of $\mathrm{RD}$ cells infected with CA16 were evaluated. $\mathrm{RD}$ cells were transfected with empty vector, pcDNA6.0-myc/HisB-MAVS plasmids for $24 \mathrm{~h}$ or an shRNA negative control and MAVS-shRNA1 plasmids for $36 \mathrm{~h}$, followed by infection with CA16 $(\mathrm{MOI}=1)$. At $12 \mathrm{~h}$ pi with CA16, the amounts of virus in the supernatant were evaluated.

ones (Fig. 6e). Next, we reduced the MAVS expression using shRNAs (MAVS-shRNA1, MAVS-shRNA2, MAVS-shRNA3, MAVS-shRNA4), resulting in a marked knockdown of MAVS, as confirmed by western blot and real-time PCR analysis (Fig. 6d). Compared with MAVS-shRNA3 and
MAVS-shRNA4, MAVS-shRNA1 and MAVS-shRNA2 can more effectively downregulate the levels of MAVS expression. Then MAVS-shRNA1 and MAVS-shRNA2 were used for further study. As shown in Fig. 6f, there was a prominent increase in CA16 replication after the expression of MAVS 
was inhibited compared with the control cells. Taken together, these results showed that overexpression of MAVS efficiently reduces the CA16 replication. To further assess the effect of overexpression or knockdown of MAVS on CA16 production, the amounts of virus in the supernatant of infected cells were measured by determining the TCID values. The cells with MAVS-overexpression showed signifi-

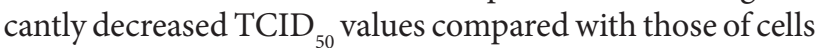
transfected with an empty vector (Fig. 6g). Meanwhile the virus titer of MAVS-knockdown cells was higher than that of cells transfected with negative control plasmid (Fig. 2g). These results suggest that MAVS is involved in the replication of CA16 to host cells.

\section{Discussion}

In this paper, we revealed that overexpression of MAVS triggers apoptosis and autophagy in RD cells. Moreover, we uncovered the mechanisms underlying the apoptosis induction and showed that MAVS induces apoptosis by activating the JNK signaling pathway and inhibiting the ERK signaling pathway. By using autophagy inhibitor and inducer, we demonstrated that autophagy inhibition could enhance MAVS-induced apoptosis. MAVS expression was also verified to be downregulated in CA16-infected RD cells and MAVS overexpression inhibited CA16 replication. In summary, our findings reveal novel functions of MAVS, which may help to provide new targets for control of CA16 infections in the future.

MAVS, a vital bridge protein, plays critical roles in the regulation of apoptosis (Seth et al., 2005). MAVS is located in mitochondria and contains a N-terminal CARD-like domain (Seth et al., 2005). The CARD, a homotypic interaction motif, participates in apoptosis signaling pathways. For example, Nod1, a CARD-containing protein, promotes caspase9-induced apoptosis (Ting et al., 2008). Ipaf contains an $\mathrm{NH} 2$-terminal CARD domain that induces the activation of caspase-1-dependent apoptosis in transfected cells (Nouri et al., 2016). It has been reported that overexpression of MAVS induced apoptosis in $293 \mathrm{~T}$ cells at $48 \mathrm{~h}$ post-transfection (Lei et al., 2009). In our study, we found that overexpression of MAVS induces apoptosis at $24 \mathrm{~h}$ post-transfection in $\mathrm{RD}$ cells. It is possible that the inconsistency of apoptotic induction time suggests that the mechanisms of MAVS-induced apoptosis may be different in different cells.

The ERK and JNK belong to the superfamily of MAP-kinases that are involved in the regulation of various biological processes including apoptosis (Johnson and Lapadat, 2002). Rajmani et al. reported that hemagglutinin neuraminidase (HN) of Newcastle disease virus upregulates the SAPK/JNK pathway, leading to transactivation of c-Jun, which in turn activates apoptosis signaling (Rajmani et al., 2015). Deng et al. demonstrated that hepatitis $\mathrm{C}$ virus (HCV) infection induces ROS/JNK signaling which leads to Bax activation and apoptosis induction (Deng et al., 2015). The ERK signaling pathway also has been reported to participate in apoptosis regulation and has been shown to have a dual role in the process (Li et al., 2014). Paeoniflorin has been shown to inhibit human pancreatic cancer cell apoptosis via suppression of ERK signaling (Yang et al., 2016). In another study, Rossi et al. revealed that adiponectin inhibits neutrophil apoptosis via activation of the ERK1/2 MAP kinase (Rossi and Lord, 2013). However, whether the JNK and ERK signaling pathways participate in MAVS-induced apoptosis remains unclear. In this study, we confirmed that ERK was inhibited and JNK was activated after MAVS overexpression, contributing to the upregulation of cleaved PARP and cleaved caspase-3. Disrupting JNK with SP600125 and ERK with PD98059 inhibited and enhanced the apoptosis response, respectively, demonstrating that overexpression of MAVS triggers apoptosis via a mechanism that involves, in part, the regulation of JNK and ERK signals.

Autophagy and apoptosis are two of the most important cellular processes that allow cells and organisms to cope with and respond to cellular damage (Mukhopadhyay et al., 2014). Autophagy and apoptosis are intrinsically linked by multiple co-regulatory signals (Mukhopadhyay et al., 2014). Accumulating evidences revealed that the functional relationship between autophagy and apoptosis is context-dependent (Mukhopadhyay et al., 2014). Autophagy can be an adaptation to avoid apoptosis and limit caspase-dependent cell death (Mukhopadhyay et al., 2014). Huang et al. demonstrated that inhibition of autophagy with 3-MA promoted apoptosis, while inhibition of apoptosis with Z-VAD-FMK facilitated autophagy in DENV2-infected HUVECs and EA.hy926 cells (Huang et al., 2014). Zhou et al. showed that inhibition of autophagy with 3-MA increased vpr-induced apoptosis (Zhou et al., 2017). In this study, we also found that autophagy inhibition promotes apoptotic cell death in RD cells. Several mechanisms may explain why autophagy inhibition enhances apoptotic cell death. One possible mechanism is that autophagy serves as a major protective mechanism that allows cells to survive in response to multiple stressors and that it helps organisms defend against degenerative, infectious, and neoplastic diseases (Kemp, 2017). Another possible mechanism is that autophagy can influence the apoptotic cascades by regulating the amount and activity of caspases and apoptosis associated proteins. For example, the autophagy-related protein 3 complex releases $\mathrm{Bcl}-2$ to block apoptotic pathways (Rubinstein et al., 2011).

MAVS may play key roles in transducing antiviral signaling and has been a prime target of viruses to evade the host immune system (Lei et al., 2009). Li et al. showed that Borna disease virus (BDV) X protein colocalizes and interacts with MAVS in the mitochondria to block programmed cell death 
(Li et al., 2013). In this study, we found that expression of MAVS was inhibited by CA16 and overexpression of MAVS could inhibit CA16 replication. It is interesting that MAVS has the specific mechanism of CA16 inhibition. Mukherjee et al. revealed that the Coxsackievirus B 3Cpro protease cleaves MAVS to attenuate host type I interferon (Mukherjee et al., 2011). Rui et al. discovered that the 3Cpro proteins from CA16 bind melanoma differentiation-associated gene 5 (MDA-5) and inhibit its interaction with MAVS (Rui et al., 2017). However, whether CA16 or viral proteins could interact with MAVS directly or by other mechanisms that were adopted to regulate CA 16 replication are questions that require further investigation.

Acknowledgments. This work was supported by the National Natural Sciences Foundation of China (No. 81641093, 81371790, 81371422, 81571481, and 81701571), the Fundamental Research Funds for Shenzhen Science and Technology Innovation Committee (No. JCYJ20170818143952175), the Central Universities of China and the Translational Medical Research Fund of Wuhan University School of Medicine, the Hubei Provincial Natural Science Foundation of China (No. 2016CFB306), and the Hubei Province Health and Family Planning Scientific Research Projects (No. WJ2017H0041).

\section{References}

Akira S, Uematsu S, Takeuchi O (2006): Pathogen recognition and innate immunity. Cell 124, 783-801. https://doi. org/10.1016/j.cell.2006.02.015

Deng L, Chen M, Tanaka M, Ku Y, Itoh T, Shoji I, Hotta H (2015): HCV upregulates Bim through the ROS/JNK signalling pathway, leading to Bax-mediated apoptosis. J. Gen. Virol. 96, 2670-2683. https://doi.org/10.1099/jgv.0.000221

Dhanasekaran DN, Reddy EP (2008): JNK signaling in apoptosis. Oncogene 27, 6245-6251. https://doi.org/10.1038/ onc. 2008.301

Huang J, Li Y, Qi Y, Zhang Y, Zhang L, Wang Z, Zhang X, Gui L (2014): Coordinated regulation of autophagy and apoptosis determines endothelial cell fate during Dengue virus type 2 infection. Mol. Cell. Biochem. 397, 157-165. https://doi.org/10.1007/s11010-014-2183-3

Johnson GL, Lapadat R (2002): Mitogen-activated protein kinase pathways mediated by ERK, JNK, and p38 protein kinases. Science 298, 1911-1912. https://doi.org/10.1126/ science. 1072682

Jones E, Pillay TD, Liu F, Luo L, Bazo-Alvarez JC, Yuan C, Zhao S, Chen Q, Li Y, Liao Q, Yu H, Rogier VDH, Sabanathan S (2018): Outcomes following severe hand foot and mouth disease: A systematic review and meta-analysis. Eur. J. Paediatr. Neurol. 22, 763-773. https://doi.org/10.1016/j. ejpn.2018.04.007

Kawai T, Akira S (2006): Innate immune recognition of viral infection. Nat. Immunol. 7, 131-137. https://doi.org/10.1038/ $\underline{\text { ni1303 }}$
Kemp MG (2017): Crosstalk Between Apoptosis and Autophagy: Environmental Genotoxins, Infection, and Innate Immunity. J. Cell. Death 9, 2114263837. https://doi. org/10.1177/1179670716685085

Lei Y, Moore CB, Liesman RM, O`Connor BP, Bergstralh DT, Chen ZJ, Pickles RJ, Ting JP (2009): MAVS-mediated apoptosis and its inhibition by viral proteins. PLoS One 4, e5466. https://doi.org/10.1371/journal.pone.0005466

Li Q, Chen M, Liu H, Yang L, Yang T, He G (2014): The dual role of ERK signaling in the apoptosis of neurons. Front Biosci. (Landmark Ed.) 19, 1411-1417. https://doi. org/10.2741/4291

Li Y, Song W, Wu J, Zhang Q, He J, Li A, Qian J, Zhai A, Hu Y, Kao W, Wei L, Zhang F, Xu D (2013): MAVS-mediated host cell defense is inhibited by Borna disease virus. Int J Biochem Cell. Biol. 45, 1546-1555. https://doi.org/10.1016/j. biocel.2013.05.012

Ma H, Su L, Yue H, Yin X, Zhao J, Zhang S, Kung H, Xu Z, Miao $J$ (2015): HMBOX1 interacts with MT2A to regulate autophagy and apoptosis in vascular endothelial cells. Sci. Rep. 5, 15121. https://doi.org/10.1038/srep15121

Mao Q, Wang Y, Yao X, Bian L, Wu X, Xu M, Liang Z (2014): Coxsackievirus A16: epidemiology, diagnosis, and vaccine. Hum. Vaccin. Immunother. 10, 360-367. https://doi. org $/ 10.4161 /$ hv. 27087

Mukherjee A, Morosky SA, Delorme-Axford E, Dybdahl-Sissoko N, Oberste MS, Wang T, Coyne CB (2011): The coxsackievirus B 3C protease cleaves MAVS and TRIF to attenuate host type I interferon and apoptotic signaling. PLoS Pathog. 7, e1001311. https://doi.org/10.1371/journal. ppat. 1001311

Mukhopadhyay S, Panda PK, Sinha N, Das DN, Bhutia SK (2014): Autophagy and apoptosis: where do they meet? Apoptosis 19, 555-566. https://doi.org/10.1007/s10495-014-0967-2

Nouri HR, Karkhah A, Mohammadzadeh I, Sankian M (2016): Elevated caspase 1 activity and IL1beta expression are associated with the IPAF inflammasome in an experimental model of allergy. Mol. Med. Rep. 13, 3356-3362. https:// doi.org/10.3892/mmr.2016.4934

Ohsumi Y (2014): Historical landmarks of autophagy research. Cell Res. 24, 9-23. https://doi.org/10.1038/cr.2013.169

Qin Y, Xue B, Liu C, Wang X, Tian R, Xie Q, Guo M, Li G, Yang D, Zhu H (2017): NLRX1 mediates MAVS degradation to attenuate hepatitis $C$ virus-induced innate immune response through PCBP2. J. Virol. 91, pii: e01264-17. https://doi.org/10.1128/JVI.01264-17

Rajmani RS, Gandham RK, Gupta SK, Sahoo AP, Singh PK, Kumar R, Saxena S, Chaturvedi U, Tiwari AK (2015): HN Protein of Newcastle Disease Virus Induces Apoptosis Through SAPK/JNK Pathway. Appl. Biochem. Biotechnol. 177, 940-956. https://doi.org/10.1007/s12010-015-1788-7

Rossi A, Lord JM (2013): Adiponectin inhibits neutrophil apoptosis via activation of AMP kinase, PKB and ERK 1/2 MAP kinase. Apoptosis 18, 1469-1480. https://doi.org/10.1007/ s10495-013-0893-8

Rubinstein AD, Eisenstein M, Ber Y, Bialik S, Kimchi A (2011): The autophagy protein Atg12 associates with antiapoptotic Bcl-2 family members to promote mitochondrial apop- 
tosis. Mol. Cell. 44, 698-709. https://doi.org/10.1016/j. molcel.2011.10.014

Rui Y, Su J, Wang H, Chang J, Wang S, Zheng W, Cai Y, Wei W, Gordy JT, Markham R, Kong W, Zhang W, Yu XF (2017): Disruption of MDA5-Mediated Innate Immune Responses by the 3C Proteins of Coxsackievirus A16, Coxsackievirus A6, and Enterovirus D68. J. Virol. 91, pii: e00546-17. https:// doi.org/10.1128/JVI.00546-17

Seth RB, Sun L, Ea CK, Chen ZJ (2005): Identification and characterization of MAVS, a mitochondrial antiviral signaling protein that activates NF-kappaB and IRF 3. Cell 122, 669-682. https://doi.org/10.1016/j.cell.2005.08.012

Shi Y, He X, Zhu G, Tu H, Liu Z, Li W, Han S, Yin J, Peng B, Liu W (2015): Coxsackievirus A16 elicits incomplete autophagy involving the mTOR and ERK pathways. PLoS One 10, e122109. https://doi.org/10.1371/journal.pone.0122109

Shi Y, Tu H, Chen X, Zhang Y, Chen L, Liu Z, Sheng J, Han S, Yin J, Peng B, He X, Liu W (2016): The long non-coding RNA expression profile of Coxsackievirus A16 infected RD cells identified by RNA-seq. Virol. Sin. 31, 131-141. https:// doi.org/10.1007/s12250-015-3693-1

Sun Q, Sun L, Liu HH, Chen X, Seth RB, Forman J, Chen ZJ (2006): The specific and essential role of MAVS in antiviral innate immune responses. Immunity 24, 633-642. https://doi. org/10.1016/j.immuni.2006.04.004

Sun X, Sun L, Zhao Y, Li Y, Lin W, Chen D, Sun Q (2016): MAVS maintains mitochondrial homeostasis via autophagy. Cell. Discov. 2, 16024. https://doi.org/10.1038/celldisc.2016.24

Ting JP, Lovering RC, Alnemri ES, Bertin J, Boss JM, Davis BK, Flavell RA, Girardin SE, Godzik A, Harton JA, Hoffman HM, Hugot JP, Inohara N, Mackenzie A, Maltais LJ, Nunez G, Ogura Y, Otten LA, Philpott D, Reed JC, Reith W, Schreiber S, Steimle V, Ward PA (2008): The NLR gene family: a standard nomenclature. Immunity $28,285-287$. https://doi.org/10.1016/j.immuni.2008.02.005
Xie BS, He XX, Ai ZL, Yao SK (2014): Involvement of beta-catenin in matrine-induced autophagy and apoptosis in WBF344 cells. Mol. Med. Rep. 9, 2547-2553. https://doi. org $/ 10.3892 / \mathrm{mmr} .2014 .2107$

Yang N, Cui H, Han F, Zhang L, Huang T, Zhou Y, Zhou J (2016): Paeoniflorin inhibits human pancreatic cancer cell apoptosis via suppression of MMP-9 and ERK signaling. Oncol. Lett. 12, 1471-1476. https://doi.org/10.3892/ ol.2016.4761

Yin DQ, Wang CB, Xiao-Zhou, Ji SX (2018): Epidemiology characteristics of human coxsackievirus A16 and enterovirus 71 circulating from 2009 to 2017 in Linyi, China. Jpn. J. Infect. Dis. 71, 470-473. https://doi.org/10.7883/yoken. JID.2018.035

Yoshii SR, Mizushima N (2017): Monitoring and Measuring Autophagy. Int. J. Mol. Sci. 18. https://doi.org/10.3390/ ijms 18091865

Yu L, Zhang X, Wu T, Su J, Wang Y, Wang Y, Ruan B, Niu X, Wu $Y$ (2017): Avian infectious bronchitis virus disrupts the melanoma differentiation associated gene 5 (MDA5) signaling pathway by cleavage of the adaptor protein MAVS. BMC Vet. Res. 13, 332. https://doi.org/10.1186/ s12917-017-1253-7

Zhou HY, Zheng YH, He Y, Chen Z, He B (2017): The role of autophagy in THP-1 macrophages resistance to HIV- vprinduced apoptosis. Exp. Cell. Res. 351, 68-73. https://doi. org/10.1016/j.yexcr.2016.12.024

Zhu G, Zheng Y, Zhang L, Shi Y, Li W, Liu Z, Peng B, Yin J, Liu W, He X (2013): Coxsackievirus A16 infection triggers apoptosis in RD cells by inducing ER stress. Biochem. Biophys. Res. Commun. 441, 856-861. https://doi.org/10.1016/j. bbrc.2013.10.142 